\title{
Suppression of congenital nystagmus
}

\author{
H. SH I B A S A KI, Y. Y A M A S H T A, A N D S M O T OM URA \\ From the Department of Neurology, Neurological Institute, Faculty of Medicine, Kyushu University, \\ Fukuoka City, Japan
}

SUMMARY Suppression of congenital nystagmus by eyelid closure was studied by electrooculography in four cases under various conditions in order to elucidate whether the suppression is caused by blocking of fixation or not. The nystagmus persisted in the light as well as in the dark provided that the eyes were kept open. Frenzel's glasses did not suppress the nystagmus except for one case in whom jerky nystagmus was attenuated. In the other cases, only voluntary lid closure suppressed the nystagmus. Passive lid closure while the patient attempted to keep eyes open did not suppress the nystagmus except in one case. Reflex eyelid closure by glabellar tap did not suppress the nystagmus in spite of the occurrence of Bell's phenomenon. Passive eyelid opening while the patient attempted to keep eyes closed, on the other hand, did not reproduce the nystagmus. The suppression of congenital nystagmus by lid closure, at least in some cases, seems to be related to the voluntary effort to keep eyes closed but not specifically to blocking of fixation.

Congenital nystagmus is usually a pendular nystagmus which is present at birth and continues throughout life (Cogan, 1967; Walsh and Hoyt, 1969; Dell'Osso et al., 1972). It is usually horizontal, but may be vertical (Forsythe, 1955). Its aetiology is unknown (Anderson, 1953; Walsh and Hoyt, 1969). It is occasionally familial (Anderson, 1953), and is frequently associated with various conditions such as decreased visual acuity (Gamble, 1934; Anderson, 1953), head nodding (Cox, 1936; Glover, 1937), and esotropia (von Noorden, 1976). It is characterised, above all, by the suppression during eyelid closure (Aschan et al., 1956; Suzuki, 1961; Forssman, 1964) and by the inversion of optokinetic nystagmus (Daroff et al., 1973; Komatsuzaki, 1975). In spite of the marked ocular oscillation, congenital nystagmus is usually associated only with slight disability such as vertigo, oscillopsia, and unsteadiness (Komatsuzaki, 1975). But its diagnosis is important in order to avoid unnecessary investigation for posterior fossa lesions such as neoplasm.

Previous categorisation of congenital nystagmus as "fixation nystagmus" was mainly based on the fact that the nystagmus is suppressed by eyelid closure (Aschan et al., 1956; Jung and Kornhuber,

Address for reprint requests : Dr H. Shibasaki, c/o Dr A. M. Halliday, The National Hospital, Queen Square, London WC1N 3BG.

Accepted 30 June 1978
1964). The purpose of the present study was to investigate whether this suppression of congenital nystagmus by lid closure is really related to the fixation block or not.

\section{Subjects}

CASE 1

A 49 year old Japanese woman was admitted to hospital because of visual impairment. She first noticed night blindness at 11 years of age, and this has been slowly progressive since then, especially since age 47 years. She has also had hearing difficulty since age 30 years. Shortly before admission, she described oscillopsia, but she was noted to have had spontaneous oscillation of eyes throughout her life since early infancy. Her elder sister was diagnosed as having pigmentary degeneration of the retina. There was no parental consanguinity.

Physical examination revealed a thin woman with no skeletal anomaly. Her intellectual function was normal. She could only detect finger movements in front of the right eye, and her left visual acuity was reduced to perception of light. Fundoscopic examination showed typical pigmentary degeneration of the retina and optic atrophy bilaterally. There was a continuous, horizontal, conjugate, pendular nystagmus of marked intensity in the primary position. The pendular nystagmus 
persisted during lateral gaze to either side, and was attenuated on upward and downward gaze. Sensorineural hearing deficit of moderate intensity was found on both sides. Neurological examination was otherwise normal. Laboratory studies including complete blood counts, urine, blood chemistry including serum triglyceride, beta-lipoprotein, and cholesterol, ECG, skull radiograph, cerebrospinal fluid (CSF), EEG, EMG, and conduction studies of the peripheral nerves were all normal.

A diagnosis of pigmentary degeneration of the retina with hearing deficit-Usher's syndrome (Montandon, 1972) - and congenital pendular nystagmus was made.

\section{CASE 2}

A 13 year old Japanese girl was admitted to hospital because of unsteady gait and vomiting. She was noted to have had spontaneous oscillation of the eyes since early infancy. Six months before admission she noted frontal headache, nausea, and oscillopsia of rapid onset at school, which subsided after a short rest. Three months later, a similar episode occurred. Several days before admission, she began feeling unsteadiness and nausea upon awakening, and vomited twice. Frontal headache developed in the evening, and subsided the next day. There was no fever, and no prodromal infection. Family history was non-contributory.

Physical examination showed a moderately impaired tandem gait and a marked, horizontal, jerky nystagmus which was usually directed towards the left in the primary position as well as on upward and downward gaze. On lateral gaze to either side, the nystagmus persisted with the rapid phase directed to the side of gaze. Corrected visual acuity was $20 / 20$ with the right eye and $20 / 25$ with the left. Speech was normal, and there were no other neurological abnormalities. The gait became more steady in a few weeks.

Laboratory tests, including blood, urine, CSF, skull radiograph, brain scan, and vertebral angiogram, were all normal. An EEG was moderately abnormal with disorganised background activity with excessive slow waves diffusely, depression of vertex sharp waves on the left hemisphere, and with occasional bursts of slow waves or spike and wave complexes bilaterally or on either hemisphere.

A diagnosis of acute cerebellar dysfunction and congenital nystagmus was made.

\section{CASE 3}

An 18 year old Japanese man was admitted to hospital because of tingling sensation of the lower extremities and tender oedema of the lower legs, which developed acutely about a month before admission. Paraesthesia was noted also in the hands two weeks later. Spontaneous oscillation of the eyes had been noted since early infancy. He had been aware of progressive decrease in visual acuity bilaterally since he was in primary school. Family history was non-contributory.

Upon admission, his blood pressure was $140 / 30$ $\mathrm{mmHg}$; a bruit was audible over the cubital artery. Pitting oedema of the legs was present. Corrected visual acuity was $20 / 50$ bilaterally. There was a spontaneous, horizontal, jerky nystagmus with the rapid phase mostly directed to the right in the primary position as well as during upward and downward gaze. The right beating jerky nystagmus was made more pronounced on right lateral gaze. On left lateral gaze, the jerky left beating nystagmus was elicited. Pain, tactile and vibratory sensations were moderately diminished in the legs and thumbs. Ankle jerks were absent bilaterally.

Erythrocyte sedimentation rate was $5 \mathrm{~mm}$ in 1 hour and $15 \mathrm{~mm}$ in 2 hours. Blood chemistry, urine, and CSF were normal. An EMG study disclosed fibrillation potentials and positive sharp waves in the distal lower limb muscles. Motor nerve conduction velocity of the peroneal nerve was normal. Cardiovascular dysfunction and sensory symptoms rapidly improved with daily administration of thiamine.

A diagnosis of beriberi neuropathy and congenital nystagmus was made.

\section{CASE 4}

A 29 year old Japanese man was admitted to hospital because of bilateral blepharoptosis and diplopia. After an upper respiratory infection lasting several days, he noted left blepharoptosis. Several days afterwards, he developed double vision, which was worse towards the evening. He had had ocular oscillations since infancy. Family history was negative.

His visual acuity was normal. There was a moderate ptosis bilaterally, and diplopia was noted on left lateral gaze. Orbicularis oculi muscles were slightly weak and so were deltoid muscles to moderate degree. These muscles showed easy fatiguability, and the edrophonium test was positive. There was a continuous, spontaneous, horizontal, jerky nystagmus more frequently directed to the right in the primary position as well as on downward gaze. The nystagmus was occasionally pendular in the primary position. Lateral gaze to either side did not alter the direction of the horizontal jerky nystagmus. Routine laboratory examinations were all normal. Thymoma was not detected radiologically. The myasthenic condition 
responded to anticholinesterase therapy, but nystagmus persisted.

The diagnosis in this case was myasthenia gravis and congenital nystagmus.

\section{Methods}

Ocular movements were recorded by electro-oculography (EOG). Cup electrodes were placed on the outer canthi to record horizontal movement, and on the glabella and the tip of nose to record vertical movement. The EOG was amplified with a 9 channel polygraph with time constant of $1.5 \mathrm{~s}$ for recording the original curve and $0.03 \mathrm{~s}$ for the velocity curve. Recording was made in the primary position of gaze, fixating a target in the central visual field, during eye movements in response to verbal command and, in cases with good visual acuity, during an eye-tracking test. The target movement, both saccadic and smooth pursuit, was produced by Hamamatsu-TV Oculomotor Stimulator HTV-C 582 and projected on a screen $1.5 \mathrm{~m}$ in front of the eyes.

Effects of various conditions on the nystagmus were evaluated while recording the EOG. First, the nystagmus was compared in light and darkness. Effects of eyelid closure and lid opening were examined in both light and dark conditions. Frenzel's glasses were applied to eliminate fixation. Alteration of the nystagmus on voluntary gaze to each direction was evaluated. In order to compare voluntary and passive eyelid closure, the eyelids were closed passively by the examiner's fingers while the patient was required to keep his eyes open. The influence of glabellar tap in provoking passive eyelid closure was investigated. Conversely, the eyelids were opened passively by the examiner's fingers while the patient was urged to keep his eyes closed. For the reflex eye movements, oculocephalic reflex, and optokinetic nystagmus were examined in all patients, and caloric response by irrigation of the external auditory canal with cold water in one patient.

\section{Results}

The EOG demonstrated spontaneous, horizontal, conjugate nystagmus in the primary position in all cases. The nystagmus was purely pendular in case 1 and jerky in the others. The frequency of nystagmus was 3-4 beats per second, and its amplitude ranged from 5 to 10 degrees. In all cases, the ocular movement was normal in all directions both on verbal command and on the eye-tracking test, although nystagmus was superimposed. The oculocephalic reflex was normal in all cases, although the horizontal oscillations of the eyes were persistent and superimposed. Optokinetic nystagmus in the horizontal direction was elicited in an inverted direction in two cases, with the rapid phase of nystagmus directed to the direction of movement of the target. In one case (case 4), optokinetic nystagmus was elicited only to the left side regardless of the direction of horizontal target movement. Vertical optokinetic nystagmus tested in one case (case 2) was normal and elicited to the direction opposite to the target movement. Optokinetic nystagmus was absent in case 1 who suffered from marked visual impairment. Cold stimulation of the right ear in case 4, in whom the spontaneous nystagmus was directed to the right, elicited caloric nystagmus with its rapid phase directed to the left, and the pre-existing jerky right beating nystagmus was eliminated during the stimulation.

Effects of various conditions tested are summarised in the Table. In all cases, spontaneous nystagmus was present in light and darkness to a similar extent if the eyes were kept open. Eyelid closure on command blocked the nystagmus in light and darkness. Frenzel's glasses attenuated the $\stackrel{\oplus}{+} \vec{N}$ frequency and amplitude of the nystagmus in only 7 one case (case 4), but did not influence the nystagmus at all in three others (Fig. 1). In these three cases, only eyelid closure on command blocked the nystagmus while Frenzel's glasses were applied. Passive eyelid opening while the nystagmus was blocked during voluntary lid closure did not reproduce the nystagmus in any of four cases (Fig. 2). On the other hand, passive lid closure while the patient was attempting to keep his eyes open did not block the nystagmus in two cases (Fig. 3). but did block it in one (case 4). Glabellar tap produced reflex lid closure associated with a transient upward deviation of the eyes (Bell's phenomenon), but the nystagmus was not influenced in the three cases tested (Fig. 4).

\section{Table Congenital nystagmus under various conditions}

\begin{tabular}{|c|c|c|c|c|c|c|}
\hline \multicolumn{3}{|c|}{ Ocular conditions } & \multicolumn{4}{|c|}{ Case number } \\
\hline Light & $\begin{array}{l}\text { Eyes } \\
\text { Fren } \\
\text { Eyes }\end{array}$ & $\begin{array}{l}\text { s open } \\
\text { nzel } \\
\text { s closed }\end{array}$ & $\begin{array}{l}\text { Present } \\
\text { Present } \\
\text { Absent }\end{array}$ & $\begin{array}{l}\text { Present } \\
\text { Present } \\
\text { Absent }\end{array}$ & $\begin{array}{l}\text { Present } \\
\text { Present } \\
\text { Absent }\end{array}$ & $\begin{array}{l}\text { Present } \\
\text { Attenuated } \\
\text { Absent }\end{array}$ \\
\hline Dark & $\begin{array}{l}\text { Eyes } \\
\text { Fren } \\
\text { Eyes }\end{array}$ & $\begin{array}{l}\text { s open } \\
\text { nzel } \\
\text { s closed }\end{array}$ & $\begin{array}{l}\text { Present } \\
\text { Present } \\
\text { Absent }\end{array}$ & $\begin{array}{l}\text { Present } \\
\text { Present } \\
\text { Absent }\end{array}$ & $\begin{array}{l}\text { Present } \\
\text { Present } \\
\text { Absent }\end{array}$ & $\begin{array}{l}\text { Present } \\
\text { Attenuated } \\
\text { Absent }\end{array}$ \\
\hline \multicolumn{3}{|c|}{ Lid closure $\begin{array}{l}\text { Voluntary } \\
\text { Passive }\end{array}$} & Blocked & $\begin{array}{l}\text { Blocked } \\
\text { Persist }\end{array}$ & $\begin{array}{l}\text { Blocked } \\
\text { Persist }\end{array}$ & $\begin{array}{l}\text { Blocked } \\
\text { Blocked }\end{array}$ \\
\hline \multicolumn{3}{|c|}{ Glabellar tap } & 一 & Persist & Persist & Persist \\
\hline $\begin{array}{l}\text { Lid } \\
\text { openin }\end{array}$ & & $\begin{array}{l}\text { Voluntary } \\
\text { Passive }\end{array}$ & $\begin{array}{l}\text { Present } \\
\text { Absent }\end{array}$ & $\begin{array}{l}\text { Present } \\
\text { Absent }\end{array}$ & $\begin{array}{l}\text { Present } \\
\text { Absent }\end{array}$ & $\begin{array}{l}\text { Present } \\
\text { Absent }\end{array}$ \\
\hline
\end{tabular}

Present $=$ congenital nystagmus seen.

Absent $=$ congenital nystagmus not seen. 
(a)

Frenzel's Spectacles in Light Room
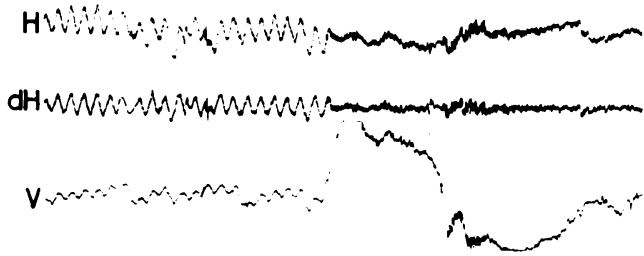

dV

EC $110^{\circ}$

(b)

Frenzel's Spectacles in Dark Room

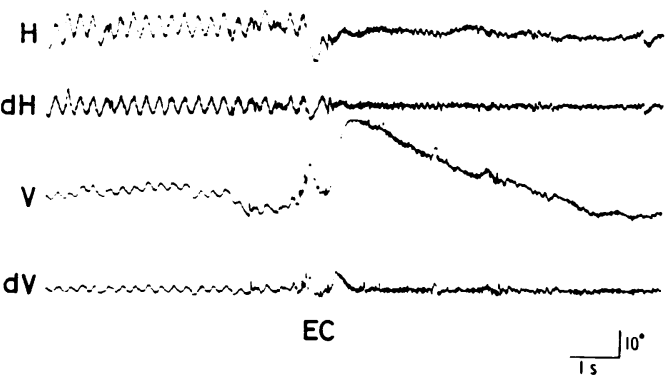

Fig. 1 Effects of application of Frenzel's glasses and of eyelid closure on pendular nystagmus $(a)$ in the light and $(b)$ in the dark in case 1. Note the suppression of nystagmus only by lid closure (EC) both in the light and dark. $H$ and $d H$ denote the horizontal displacement and velocity curves respectively, and $V$ and $d V$ indicate the vertical displacement and velocity curves respectively. Upward deflection shows the leftward movement for the horizontal and the upward movement for the vertical component.

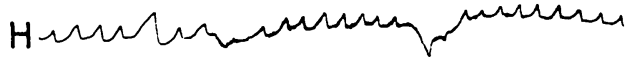

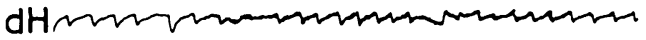

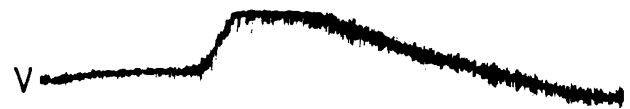

$d V$

Passive EC $120^{\circ}$

Fig. 3 Effect of passive eyelid closure on jerky nystagmus in case 3. Passive lid closure (passive EC) does not suppress the right beating jerky nystagmus. Note upward deviation of eyes (Bell's phenomenon) at the time of passive lid closure.

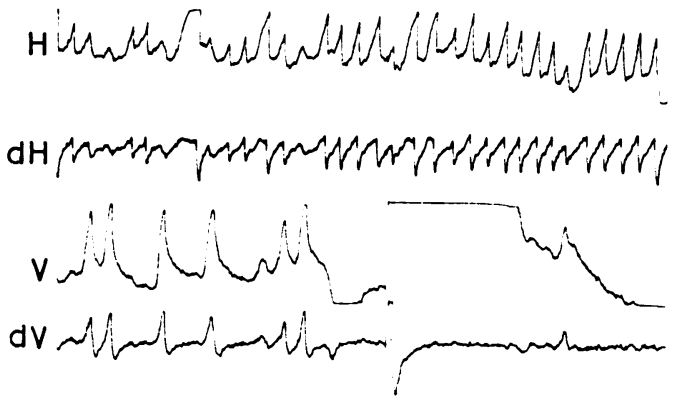

Glabellar Tap

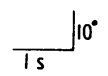

Fig. 4 Effect of glabellar tap on jerky nystagmus in case 3. Nystagmus persists in spite of the involuntary eyelid closure as a result of a glabellar reflex.
Fig. 2 Effect of passive eyelid opening on pendular nystagmus in case 1 . The nystagmus is suppressed during lid closure $(E C)$, and does not reappear on passive opening of the eyelid (passive EO).
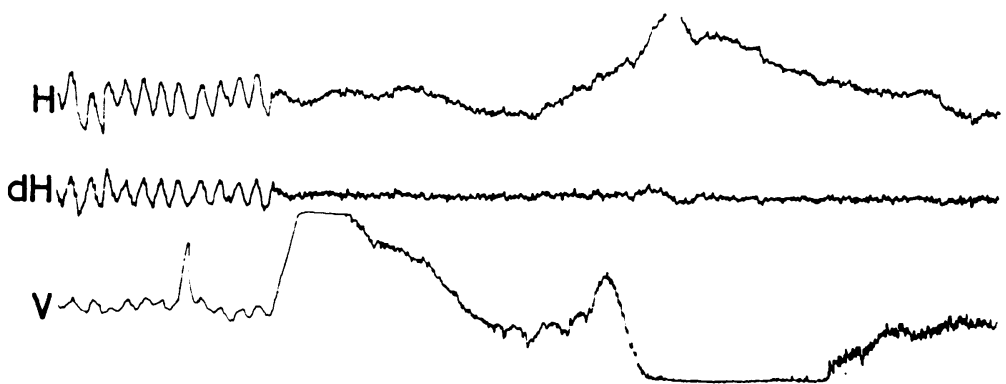

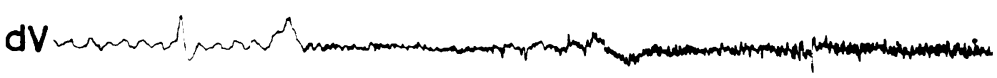




\section{Discussion}

Suppression by eyelid closure has been considered to be one of the main features of congenital nystagmus (Aschan et al., 1956; Suzuki, 1961; Forssman, 1964; Jung and Kornhuber, 1964), and been used in differentiating it from vestibular nystagmus (Komatsuzaki, 1975). It has been ascribed primarily to the blocking of fixation (Aschan et al., 1956). Hence the congenital nystagmus was put into a category of "fixation nystagmus." There seem to be, however, at least five possible factors which would explain its suppression by lid closure. These include elimination of the light stimulation, proprioceptive impulses from the orbicularis oculi muscles, voluntary effort to close or keep the eyes closed, strong and persistent upward deviation of eyes associated with lid closure or Bell's phenomenon, and actual blocking of fixation.

In the present study, the nystagmus persisted both in the light and in the dark so long as the eyes were kept open. Suzuki (1961) found that the pendular nystagmus increased by lid opening either in the dark or in the light, and threw some doubt on the role of fixation in facilitating this kind of nystagmus. Forssman (1964) studied a large number of cases with congenital nystagmus and found that, in the dark, the nystagmus was present to a variable extent if the eyes were kept open. On the other hand, the nystagmus was almost always suppressed when the eyes were closed. In the present study, the nystagmus persisted whether in the light or in the dark, and only voluntary eyelid closure suppressed it. Therefore, the mechanism of lid closure in suppressing the congenital nystagmus does not seem to be related uniquely to an effect of eliminating the light stimulation.

Application of Frenzel's glasses to prevent fixation did not influence the nystagmus to any noticeable degree except for one case. This fact does not support the theory of "fixation" being the main trigger mechanism for congenital nystagmus, although there seem to be exceptions such as case 4 of the present series. Aschan et al. (1956) studied one case of congenital nystagmus with Frenzel's glasses and found no suppression of the nystagmus. They ascribed their result to a failure of Frenzel's glasses to block fixation. But in the present study, wearing Frenzel's glasses did not alter the nystagmus even in the darkness.

Moreover, involuntary closure of eyelids such as passive lid closure, while the patient was attempting to keep his eyes open, blocked the nystagmus in one case (case 4) but did not affect it in the two others tested. Glabellar tap did not suppress the nystagmus although Bell's phenomenon could be demonstrated. These facts do not support the theory that suppression of the nystagmus by lid closure is caused by proprioceptive impulses or a reflex mechanism. It was also found in the present study that passive opening of the eyelids, while the patient was attempting to keep his eyes closed, could not reproduce the nystagmus in any of the four cases. It is conceivable, from these findings, that, at least in some forms of congenital nystagmus, its suppression by eyelid closure is related directly to the voluntary effort to keep eyes closed but not to actual blocking of the fixation. Case 4 of the present series is considered to have, at least in part, a feature of "fixation nystagmus" because it was suppressed by Frenzel's glasses and by passive eyelid closure. Arkin et al. (1972) studied eight cases of congenital nystagmus by EOG during wakefulness as well as during sleep, and found that clear nystagmus was not discernible either during non-REM sleep or REM sleep. They stressed the lack of nystagmus during REM sleep, in which the eyes move as if tracking moving objects.

Strong and persistent upward deviation of the $\stackrel{\oplus}{\rightarrow}$ eyes accompanying voluntary lid closure might $\overrightarrow{0} \vec{\circ}$ play an important role in suppressing the con- 0 genital nystagmus. In fact, an extreme upward gaze on command suppressed the nystagmus in $\underset{\mathbb{Q}}{\circ}$ some cases of the present series. But involuntary eyelid closures with a marked Bell's phenomenon (Figs. 3 and 4) did not suppress the nystagmus. On the other hand, even the slightest eyelid closure suppressed the nystagmus. These facts seem to be against the hypothesis that the Bell's phenomenon itself is causing the suppression of the nystagmus.

The aetiology of congenital nystagmus is unknown (Anderson, 1953; Walsh and Hoyt, 1969). Some cases show familial occurrence (Glover, 1937; Anderson, 1953). Sites of lesion responsible for congenital nystagmus, whether cortical, subcortical, or vestibular, remain entirely unsolved (Anderson, 1953). Defect in sensory vision is not believed to be the primary pathophysiological mechanism (Cogan, 1967; Dell'Osso et al., 1972). Jung and Kornhuber (1964) attributed congenital fixation nystagmus to dysfunction of the brain stem oculomotor centre. Dell'Osso et al. (1972) incriminated an abnormality in the control system for eye movements, and proposed a pathophysiological defect in the generation of saccadic movement with a compensating reliance on the smooth pursuit system, and possibly a further generation of instability in the latter system as a cause of the pendular nystagmus. Daroff et al. (1973) found that a vertical line suppressed a horizontal pendular oscillation of eyes in two patients with con- 
genital nystagmus, and proposed a hypothesis of preferential cortical development of vertical line detectors as a basis of this phenomenon. The pathophysiology of congenital nystagmus remains to be elucidated but, at least in some cases of congenital nystagmus, fixation does not appear to be playing an important role.

\section{References}

Anderson, J. R. (1953). Causes and treatment of congenital eccentric nystagmus. British Journal of Ophthalmology, 37, 267-281.

Arkin, A. M., Lutzky, H., and Toth, M. F. (1972). Congenital nystagmus and sleep: a replication. Psychophysiology, 9, 210-217.

Aschan, G., Bergstedt, M., and Stahle, J. (1956). Nystagmography. Recording of nystagmus in clinical neuro-otological examinations. Acta Otolaryngologica, Supplement 129, 1-103.

Cogan, D. G. (1967). Congenital nystagmus. Canadian Journal of Ophthalmology, 2, 4-10.

Cox, R. A. (1936). Congenital head-nodding and nystagmus. Report of a case. Archives of Ophthalmology, 15, 1032-1036.

Daroff, R. B., Hoyt, W. F., Bettman, J. W., Jr., and Lessell, S. (1973). Suppression and facilitation of congenital nystagmus by vertical lines. Neurology (Minneapolis), 23, 530-533.

Dell'Osso, L., Gauthier, G., Liberman, G., and Stark, L. (1972). Eye movement recordings as a diagnostic tool in a case of congenital nystagmus. American Journal of Optometry and Physiological Optics, 49, 2-13.
Forssman, B. (1964). A study of congenital nystagmus. Acta Otolaryngologica, 57, 427-449.

Forsythe, W. I. (1955). Congenital hereditary vertical nystagmus. Journal of Neurology, Neurosurgery, and Psychiatry, 18, 196-198.

Gamble, R. C. (1934). The visual prognosis for children with congenital nystagmus. A statistical study. Transactions of the American Ophthalmological Society, 32, 485-496.

Glover, L. P. (1937). Congenital nystagmus. Archives of Ophthalmology, 17, 705-706.

Jung, R., and Kornhuber, H. H. (1964). Results of electronystagmography in man: the value of optokinetic, vestibular, and spontaneous nystagmus for neurologic diagnosis and research. In The Oculomotor System, pp. 428-482. Edited by M. B. Bender. Hoeber: New York.

Komatsuzaki, A. (1975). The diagnostic significance of nystagmus and the abnormal eye movements. Brain and Development (Tokyo), 7, 2-16.

Montandon, A. (1972). Usher syndrome. In Handbook of Clinical Neurology. Vol. 13, pp. 441-450. Neuroretinal Degeneration. Edited by P. J. Vinken and G. W. Bruyn. North-Holland: Amsterdam.

Suzuki, J. (1961). A study on pendular nystagmus. Pendular nystagmus: its contribution to the understanding of nystagmus mechanisms. Acta Otolaryngologica, 53, 381-390.

von Noorden, G. K. (1976). The nystagmus compensation (blockage) syndrome. American Journal of Ophthalmology, 82, 283-290.

Walsh, F. B., and Hoyt, W. F. (1969). Clinical Neuroophthalmology. Third edition, vol. 1, pp. 279-281. Williams and Wilkins: Baltimore. 